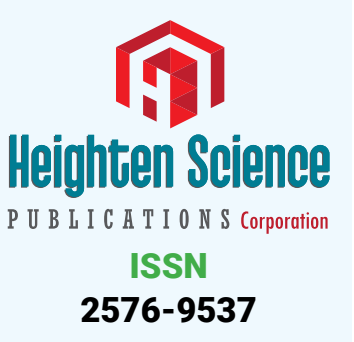

*Address for Correspondence: Praroop Gupta, Post Graduate Resident, Department Of Surgery, UCMS and GTB Hospital, Delhi, India, Tel: +919013823154; Email: praroopgupta@gmail.com Submitted: 06 August 2018 Approved: 13 August 2018 Published: 14 August 2018 Copyright: @ 2018 Agrawal V, et al. This is an open access article distributed under the Creative Commons Attribution License, which permits unrestricted use, distribution, and reproduction in any medium, provided the original work is properly cited

Keywords: Gossypiboma; Retained Surgical Sponge; Transmural migration

Abbreviations: CECT: Contrast Enhanced Computed Tomography; CT: Computed Tomography; MRI: Magnetic Resonance Imaging RSS: Retained Surgical Sponge; UGIE: Upper Gastrolntestinal Endoscop

\section{Gossypiboma due to a retained surgical sponge following abdominal hysterectomy, complicated by intestinal migration and small bowel obstruction- A Case Report}

\author{
Vivek Agrawal ${ }^{1}$ and Praroop Gupta ${ }^{2 *}$ \\ 'Director Professor, Department Of Surgery, UCMS and GTB Hospital, Delhi, India \\ ${ }^{2}$ Post Graduate Resident, Department Of Surgery, UCMS and GTB Hospital, Delhi, India
}

\section{Abstract}

A gossypiboma is a mass of cotton material from any source, left in a body cavity after a surgical procedure. This enhances the morbidity, cost of treatment and potential mortality to the patient with the addition of medicolegal issues. We report a case of a 32 year old lady who presented with complaints of central abdominal pain and vomiting for 1 month, fever for 20 days and non-passage of flatus and faeces for 5 days. She had undergone a total abdominal hysterectomy 4 months prior. On clinical examination, adhesive small intestinal obstruction was suspected. On CECT evaluation, a gossypiboma was suggested to have possibly migrated into the small bowel. Laparotomy revealed the presence of clumped bowel loops, which on dissection got torn and showed a gauze like material within the bowel lumen. A diagnosis of gossypiboma with intestinal migration of a retained surgical sponge was ascertained. The possibility of a gossypiboma, particularly in previously operated cases, must be kept in mind and measures must be taken to prevent such incidences.

\section{Introduction}

Gossypiboma is a mass comprising of cotton matrix within the body left accidentally during a surgical procedure. The body may react to this foreign body through an exudative inflammatory reaction with formation of an abscess, or an aseptic fibrotic reaction in order to encapsulate the cotton material and result into a mass [1]. This iatrogenic but avoidable misfortune, often under-reported, has damaging effects upon the health of patients, and entails embarrassment as well as medico-legal consequences $[2,3]$. The incidence of retained foreign bodies during surgery is estimated to be 1 in 5500 operations [4]. A variety of objects have been reported to be left in the abdominal cavity like surgical sponges, haemostatic forceps and pieces of drainage tubes $[3,5]$. Due to its small size, common usage and ill-defined shape, surgical sponges is the commonest to be left inside [5].

Within the abdomen, the sponge is surrounded by omentum and intestines, which attempt to encapsulate it. Transmural migration of a RSS (Retained Surgical Sponge) is a rare event. It occurs as a result of the RSS abutting against the wall of the intestine and causing erosion of the bowel loops leading to pressure necrosis and transmural migration in an attempt by the body to self-extrude the foreign body through the rectum [2]. This may also cause obstruction or fistulation. Migration of RSS has been reported to occur into many organs with ileum being most commonly involved $[3,6]$. Gossypiboma, may pose a serious diagnostic dilemma and should be considered in patients with intra-abdominal mass with prior history of surgery. Abdominal x-ray, 
ultrasonography, CT, MRI can all be used to assist in diagnosis. Surgical removal is the recommended treatment option in these cases. We report a case of a gossypiboma due to a RSS that underwent a complete transmural migration to subsequently cause small intestinal obstruction.

\section{Case Presentation}

A 32 year old lady presented with complaints of dull aching, continuous central abdominal pain and non-bilious vomiting for 1 month, fever for 20 days and nonpassage of flatus and faeces for 5 days. She had undergone an abdominal hysterectomy 4 months prior following which, on postoperative day 3 , she started complaining of abdominal discomfort which was attributed to surgery and she was discharged on postoperative day 5. At presentation, her vital parameters were stable. Examination revealed a distended abdomen, with tenderness in the umbilical and hypogastric regions and a firm large intra-abdominal lump with ill-defined margins in the lower abdomen. Bowel sounds were exaggerated and per rectal examination was unremarkable. Routine laboratory investigation results were within normal limits. Ultrasonography suggested only mild interbowel free fluid. She was admitted with the impression of adhesive small intestinal obstruction.

CECT suggested a well-defined heterogenous mixed density lesion of $8.5 \times 7.5$ cms with mottled air lucency, enhancing wall and linear hyperdense foci in the mid abdomen surrounded by the bowel loops and adhered to anterior abdominal wall. Extension into the bowel lumen was also reported. An impression of gossypiboma with migration into the small bowel was made (Figure 1). At laparotomy, dense adhesions were present between the parietal wall and the clumped bowel loops which extended from 1.3 metres distal to the duodenojejunal junction to $30 \mathrm{~cm}$ proximal to the ileocaecal junction (Figure 2a). During dissection intestinal tears occurred to reveal a gauze like material within the jejunal lumen (Figure 2b). A diagnosis of gossypiboma due to RSS was confirmed. Adhesions were released and maximum length of bowel salvaged. The clumped bowel segments were resected and bowel continuity was restored. The patient did well postoperatively.

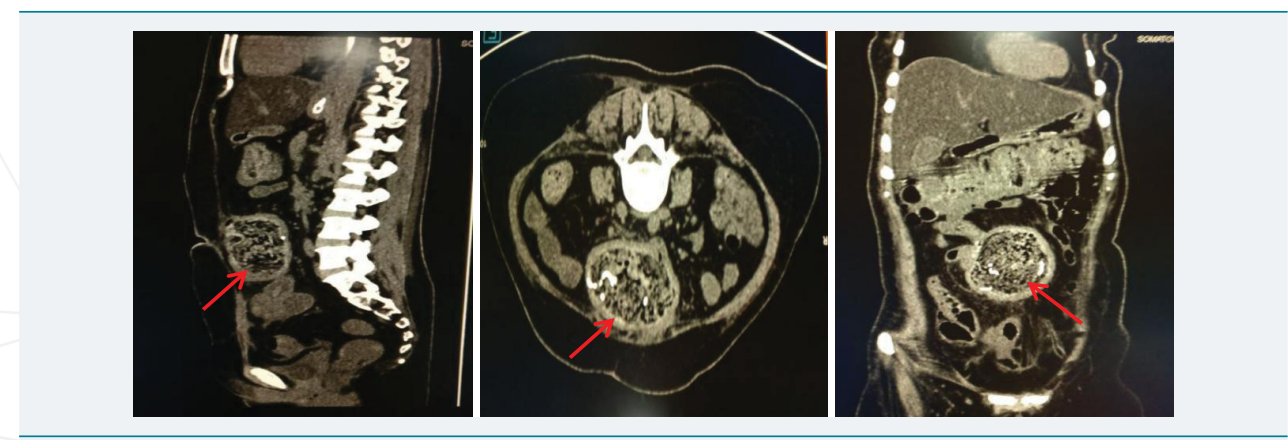

Figure 1: CECT films showing heterogenous mixed density mass (arrows) suggesting Gossypiboma.

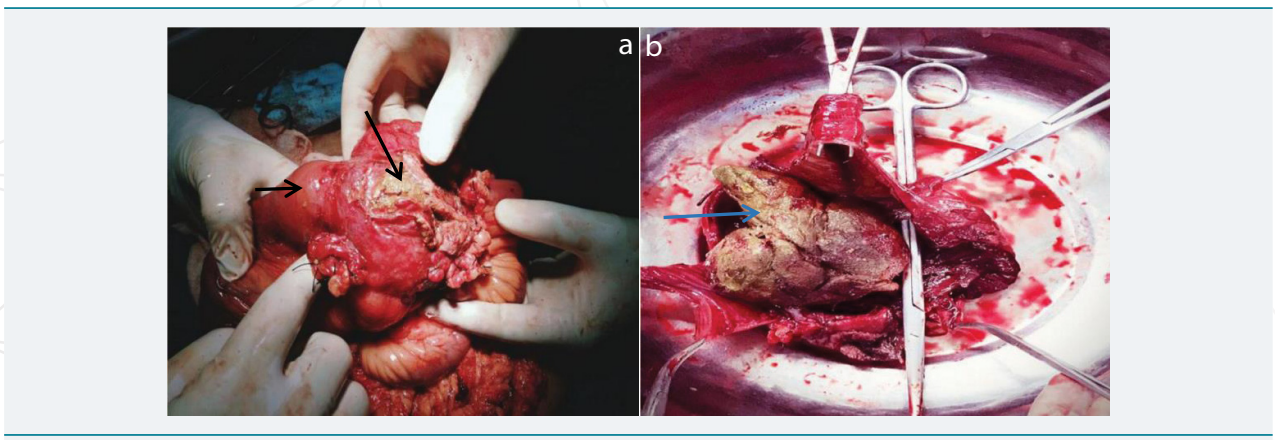

Figure 2: Intraoperative pictures showing (a) clumped bowel loops (small arrow) with a gauze like material (larger arrow) in between them, (b) opening the bowel loops revealed transmural migration of the sponge (blue arrow). 


\section{Discussion}

The first case of RSS was described by Wilson in 1884. The incidence is estimated to be 1 in 5500 surgeries [4]. The abdomen is the most common site (56\%), followed by the pelvis (18\%) and the thorax (11\%) [2]. Intraluminal migration of the RSS, as in this case, may be driven by peristaltic waves which attempt to expel it per rectally [5]. However, in most cases the RSS gets stuck at the terminal ileum causing intestinal obstruction [2]. The high index of suspicion in a patient giving a previous history of surgery and presenting with persistent abdominal pain, signs of infection or a palpable mass are diagnostic aids [2], yet many are found only at laparotomy [5]. The radio-opaque markers on sponge disintegrate with the passage of time adding to the diagnostic difficulty [5]. The most characteristic appearance on CECT is a low density heterogeneous mass with a spongiform pattern that contains gas bubbles [2] as seen in our patient (Figure1). Surgical removal (open) is the only viable option for management of RSS in most cases. Laparoscopic approach is also a possibility for those who are diagnosed early. The most common risk factors associated with RSS include unplanned changes in the surgical procedures, emergency operations, lengthy procedures and change in nursing staff during procedures and failure to count surgical instruments and sponges [6].

\section{Conclusion}

The present case highlights that one must be aware of the risk factors that could lead to a gossypiboma and proper measures must be taken to prevent them. Gossypibomas are uncommon, mostly asymptomatic and difficult to diagnose and carry medicolegal complications. It should be included in the differential diagnosis of soft-tissue masses detected in patients with history of a prior operation. Surgical removal is curative, though postoperative disease course can be morbid.

\section{References}

1. Gibbs VC, Coakley FD, Reines HD. Preventable errors in the operating room: retained foreign bodies after surgery. Curr Probl Surg. 2007; 44: 281-337. Ref.: https://tinyurl.com/yblk5wxz

2. SP Stawicki, DC Evans, J Cipolla, MJ Seamon, JJ Lukaszczyk, et al. Retained surgical foreign bodies: a comprehensive review of risks and preventive strategies. Scand J Surg. 2009; 98: 8-17. Ref.: https://tinyurl.com/y83z5hj5

3. Malhotra MK. Migratory Surgical Gossypiboma - Cause of iatrogenic perforation: Case report with review of literature. Niger J Surg. 2012; 18: 27-29. Ref.: https://tinyurl.com/yd5q8mna

4. Zhou $\mathrm{Y}$, Chen $\mathrm{P}$, Qiao $\mathrm{T}$, Chen $\mathrm{YF}$, Zong $\mathrm{L}$. Complete transmural migration of a retained surgical sponge: an atypical case in image mimicking intussusception: A case report. Medicine (Baltimore). 2017; 96: e8246. Ref.: https://tinyurl.com/y732jn3z

5. Md Moniruzzaman Sarker, AKM Golam Kibari, Md Manzurul Haque, Kali Prosad Sarker, Md Khalilur Rahman. Spontaneous transmural migration of a retained surgical mop into the small intestinal lumen causing sub-acute intestinal obstruction: a case report. TAJ. 2006; 19: 34-37. Ref.: https://tinyurl.com/y9mvdozm

6. Yun-Xiao Lv, Cheng-Chan Yu, Chun-Fang Tung, Cheng-Chung Wu. Intractable duodenal ulcer caused by transmural migration of gossypiboma into the duodenum-a case report and literature review. BMC Surgery. 2014; 14: 36. Ref.: https://tinyurl.com/yaoo3xex 